\title{
Complete systems of recursive integrals and Taylor series for solutions of Sturm-Liouville equations
}

\author{
Vladislav V. Kravchenko ${ }^{1}$, Samy Morelos ${ }^{1}$, Sébastien Tremblay ${ }^{2}$ \\ ${ }^{1}$ Departamento de Matemáticas, CINVESTAV del IPN, Unidad \\ Querétaro, Libramiento Norponiente No. 2000 C.P. 76230 Fracc. \\ Real de Juriquilla, Querétaro, Mexico \\ ${ }^{2}$ Département de mathématiques et d'informatique, Université du \\ Québec, Trois-Rivières, Québec, G9A 5H7, Canada
}

October 11, 2018

\begin{abstract}
Consider an arbitrary complex-valued, twice continuously differentiable, nonvanishing function $\varphi$ defined on a finite segment $[a, b] \subset \mathbb{R}$. Let us introduce an infinite system of functions constructed in the following way. Each subsequent function is a primitive of the preceding one multiplied or divided by $\varphi$ alternately. The obtained system of functions is a generalization of the system of powers $\left\{\left(x-x_{0}\right)^{k}\right\}_{k=0}^{\infty}$. We study its completeness as well as the completeness of its subsets in different functional spaces. This system of recursive integrals results to be closely related to so-called $L$-bases arising in the theory of transmutation operators for linear ordinary differential equations.

Besides the results on the completeness of the system of recursive integrals we show a deep analogy between the expansions in terms of the recursive integrals and Taylor expansions. We prove a generalization of the Taylor theorem with the Lagrange form of the remainder term and find an explicit formula for transforming a generalized Taylor expansion of a function in terms of the recursive integrals into a usual Taylor expansion. As a direct corollary of the formula we obtain the following new result concerning solutions of the Sturm-Liouville equation. Given a regular nonvanishing complex valued solution $y_{0}$ of the equation $y^{\prime \prime}+q(x) y=0, x \in(a, b)$, assume that it is $n$ times differentiable at a point $x_{0} \in[a, b]$. We present explicit formulas for calculating the first $n$ derivatives at $x_{0}$ for any solution of the equation $u^{\prime \prime}+q(x) u=\lambda u$. That is, an explicit map transforming the Taylor expansion of $y_{0}$ into the Taylor expansion of $u$ is constructed.
\end{abstract}

AMS subject classification: 34B24; 41A30; 42A65

Keywords: complete system of functions; Sturm-Liouville problem

\section{Introduction}

In the recent work [16] by means of pseudoanalytic function theory [2, [17] a representation for solutions of Sturm-Liouville equations in terms of spectral parameter power series was obtained. For a proof not requiring pseudoanalytic functions we refer to [17] and [19]. The 
representation turned to be an appropriate tool for solving different Sturm-Liouville and related problems [3], [5], 6], [11], [12], [13], 14], [15], 19], 20] and [21]. As is well known (see, e.g., [23]) under certain regularity conditions a solution to the initial value problem for the Sturm-Liouville equation is an analytic function of the spectral parameter and hence admits a Taylor series expansion in powers of the spectral parameter. In fact in [16] the recursive formulas for calculating the Taylor coefficients in that expansion were proposed. The Taylor coefficients are naturally functions of the independent variable. Considered as an infinite system of functions they form a so-called $L$-basis related to a linear differential operator $L$. The theory of $L$-bases and $L$-analytic functions was developed in [9] (unfortunately that interesting and important book has not been translated into English) in relation with the concept of the operators of transmutation (see, e.g., [1], 4], 26] and the recent review [25]), also called in Russian bibliography operators of transformation (see, e.g., [22], 24]).

In [9] it was shown that to every (regular) linear ordinary differential operator one can associate a linear space spanned on an $L$-basis, and the equivalence of those linear spaces corresponding to operators of the same order was proved. Then the operator of transmutation can be regarded as an operation which transforms functions from one such linear space corresponding to a certain operator $L$ to functions from another linear space corresponding to another operator $M$, and the transformation consists in substituting the $L$-basis with the $M$-basis keeping the same coefficients in the expansion. In the recent work [18] it was shown that the situation with the linear space generated by the $L$-basis is in a sense simpler and more natural. Namely, the $L$-basis corresponding to a regular Sturm-Liouville operator on a finite interval $(a, b)$ is complete in $L_{2}(a, b)$. Moreover, in the present work we prove that in the space of piecewise continuously differentiable functions on $[a, b]$ the $L$-basis is complete with respect to the maximum norm (see theorem 9). We show a deep analogy of the $L$-basis with the system $\left\{\left(x-x_{0}\right)^{n}\right\}_{n=0}^{\infty}$ of powers of the independent variable. The formulas for calculating coefficients in the expansion of a given function in terms of those generalized powers resulted to be direct generalizations of the formulas for Taylor coefficients. We prove a generalization of the Taylor theorem with the Lagrange form of the remainder term.

A natural question then is about a relation between the generalized Taylor coefficients of a function and its ordinary Taylor coefficients. This question has not been studied previously. We find this relation and use it to obtain the main result of this paper, the representation for ordinary Taylor coefficients of any solution of the Sturm-Liouville equation $u^{\prime \prime}+q(x) u=\lambda u$ in terms of the Taylor coefficients of a particular solution $y_{0}$ of the equation $y^{\prime \prime}+q(x) y=0$. More precisely we obtain the Taylor coefficients of the quotient $u / y_{0}$. It is interesting to notice that the form of the map transforming one set of Taylor coefficients into the other is

independent of the particular form of $y_{0}$. The paper ends with examples of application of the presented result.

\section{Definition and example}

Let $f \in C^{2}(a, b) \cap C^{1}[a, b]$ be a complex valued function and $f(x) \neq 0$ for any $x \in[a, b]$. The interval $(a, b)$ is supposed to be finite. Let us consider the following auxiliary functions

$$
\widetilde{X}^{(0)}(x) \equiv X^{(0)}(x) \equiv 1,
$$




$$
\begin{aligned}
& \widetilde{X}^{(n)}(x)=n \int_{x_{0}}^{x} \widetilde{X}^{(n-1)}(s)\left(f^{2}(s)\right)^{(-1)^{n-1}} \mathrm{~d} s, \\
& X^{(n)}(x)=n \int_{x_{0}}^{x} X^{(n-1)}(s)\left(f^{2}(s)\right)^{(-1)^{n}} \mathrm{~d} s,
\end{aligned}
$$

where $x_{0}$ is an arbitrary fixed point in $[a, b]$. We introduce the infinite systems of functions

$$
\left\{f_{n}\right\}_{n=1}^{\infty} \quad \text { and } \quad\left\{g_{n}\right\}_{n=1}^{\infty}
$$

defined by the relations

$$
f_{n}=f \widetilde{X}^{(2(n-1))} \quad \text { and } \quad g_{n}=f X^{(2 n-1)} .
$$

In [18] it was shown that both systems of functions in (4) are complete in $L_{2}(a, b)$ when the point $x_{0}$ coincides with one of the end points of the interval, and if $x_{0}$ is an interior point of the interval, then the completeness of the union of two systems $\left\{f_{n}\right\}_{n=1}^{\infty} \cup\left\{g_{n}\right\}_{n=1}^{\infty}$ can be guaranteed.

The question about the completeness of the systems (44) is natural due to the following observation.

Example 1 Let $f \equiv 1, a=0, b=1$. Then it is easy to see that choosing $x_{0}=0$ we have

$$
f_{1}(x)=1, \quad f_{2}(x)=x^{2}, \quad f_{3}(x)=x^{4}, \ldots
$$

and

$$
g_{1}(x)=x, \quad g_{2}(x)=x^{3}, \ldots
$$

As is well known due to the Müntz theorem (see, e.g., [8, p. 270]) both systems of polynomials are complete in $L_{2}(0,1)$. The systems (4) represent a direct generalization of the systems of polynomials (6) and (7) if instead of $f \equiv 1$ an arbitrary sufficiently smooth and nonvanishing function is chosen.

The completeness of the system (6) can be regarded as a corollary of the completeness of the system of eigenfunctions of the Sturm-Liouville problem

$$
\begin{gathered}
u^{\prime \prime}+\lambda u=0, \quad 0<x<1, \\
u^{\prime}(0)=u^{\prime}(1)=0 .
\end{gathered}
$$

Indeed, the eigenfunctions of this regular Sturm-Liouville problem have the form $u_{n}(x)=$ $\cos n \pi x, n=0,1,2, \ldots$, and as each of them admits a uniformly convergent Taylor expansion in even powers of $x$, the system (6) is also complete.

Analogously, considering (8) with the boundary conditions $u(0)=u(1)=0$ we arrive at the complete system of eigenfunctions $v_{n}(x)=\sin n \pi x, n=1,2, \ldots$ Each of them admits a uniformly convergent Taylor expansion in odd powers of $x$ and hence the system (7) is complete in $L_{2}(0,1)$ as well. 


\section{Completeness of the systems of recursive integrals}

We will need the following theorem from [16] (for additional details see [17] and [19]) establishing the relation of the systems of functions (4) to Sturm-Liouville equations.

Theorem 2 [16] Let $q$ be a continuous complex valued function of an independent real variable $x \in[a, b], \lambda$ be an arbitrary complex number. Suppose there exists a solution $f$ of the equation

$$
f^{\prime \prime}+q f=0
$$

on $(a, b)$ such that $f \in C^{2}(a, b)$ together with $1 / f$ are bounded on $[a, b]$. Then the general solution of the equation $u^{\prime \prime}+q u=\lambda u$ on $(a, b)$ has the form

$$
u=c_{1} u_{1}+c_{2} u_{2}
$$

where $c_{1}$ and $c_{2}$ are arbitrary complex constants,

$$
u_{1}=f \sum_{k=0}^{\infty} \frac{\lambda^{k}}{(2 k) !} \tilde{X}^{(2 k)} \quad \text { and } \quad u_{2}=f \sum_{k=0}^{\infty} \frac{\lambda^{k}}{(2 k+1) !} X^{(2 k+1)}
$$

with $\widetilde{X}^{(n)}$ and $X^{(n)}$ being defined by (1)-(3) and both series converge uniformly on $[a, b]$.

Remark 3 It is easy to see that by definition the solutions $u_{1}$ and $u_{2}$ satisfy the following initial conditions

$$
\begin{gathered}
u_{1}\left(x_{0}\right)=f\left(x_{0}\right), \quad u_{1}^{\prime}\left(x_{0}\right)=f^{\prime}\left(x_{0}\right), \\
u_{2}\left(x_{0}\right)=0, \quad u_{2}^{\prime}\left(x_{0}\right)=1 / f\left(x_{0}\right) .
\end{gathered}
$$

Remark 4 It is worth mentioning that in the regular case the existence and construction of the required $f$ presents no difficulty. Let $q$ be real valued and continuous on $[a, b]$. Then (10) possesses two linearly independent regular solutions $v_{1}$ and $v_{2}$ whose zeros alternate. Thus one may choose $f=v_{1}+i v_{2}$.

Theorem 5 [18] Let $(a, b)$ be a finite interval and $f \in C^{2}(a, b) \cap C^{1}[a, b]$ be a complex valued function such that $f(x) \neq 0$ for any $x \in[a, b]$. Then both systems of functions (4) defined by the relations (5) and (11)-(3) with $x_{0}=a$ are complete in $L_{2}(a, b)$.

Proof. Under the conditions of the theorem, on the interval $(a, b)$ the function $f$ is a regular solution of the equation (10) with $q:=-f^{\prime \prime} / f$ being a continuous complex valued function. Let us consider the equation

$$
u^{\prime \prime}+q u=\lambda u
$$

with the boundary conditions

$$
u(a)=u(b)=0 .
$$

It is known (see [24, p. 36]) that the system of all eigenfunctions and generalized eigenfunctions of this problem is complete in $L_{2}(a, b)$. Due to theorem 2 and remark 3 if $\lambda_{n}$ is an eigenvalue of the problem (14), (15) then the corresponding eigenfunction up to a constant factor must coincide with $u_{2}$ from (11) where $\lambda=\lambda_{n}$, i.e., it admits a uniformly convergent 
series expansion in terms of the system of functions $g_{n}$ from (5). Moreover, if the multiplicity of $\lambda_{n}$ is greater than 1 then the corresponding generalized eigenfunctions are obtained differentiating the eigenfunction with respect to the spectral parameter $\lambda$ (see [24, p. 27]). The representation (11) of $u_{2}$ shows us that the result of this operation will be again a series in terms of the functions $g_{n}$. Thus, the eigenfunctions and the generalized eigenfunctions of the problem (14), (15) can be represented as uniformly convergent series in terms of the system of functions $\left\{g_{n}\right\}_{n=1}^{\infty}$. Then due to the Lauricella theorem about the transitivity of the property of completeness (see, e.g., [8, p. 264]) we obtain that the system $\left\{g_{n}\right\}_{n=1}^{\infty}$ is complete in $L_{2}(a, b)$.

In a similar way the completeness of $\left\{f_{n}\right\}_{n=1}^{\infty}$ is proved by considering the Sturm-Liouville problem for equation (14) with the boundary conditions

$$
f^{\prime}(a) u(a)-f(a) u^{\prime}(a)=u(b)=0 .
$$

All eigenfunctions of this problem coincide with $u_{1}$ from (11) where $\lambda=\lambda_{n}$ (this is due to remark 3) and generalized eigenfunctions are obtained from the eigenfunctions by differentiation with respect to $\lambda$. Thus, by analogy with the previously considered case all the eigenfunctions and generalized eigenfunctions of this Sturm-Liouville problem are represented as uniformly convergent series in terms of $f_{n}$, and by the Lauricella theorem $\left\{f_{n}\right\}_{n=1}^{\infty}$ is complete in $L_{2}(a, b)$.

In this theorem we assumed that the point $x_{0}$ coincided with one of the end points of the interval $(a, b)$. When $x_{0}$ is an interior point of the interval in general the systems $\left\{f_{n}\right\}_{n=1}^{\infty}$ and $\left\{g_{n}\right\}_{n=1}^{\infty}$ separately are not complete. It is easy to see on the considered above example 1 that if $a=-1$ and all other values remain unchanged then the system (6) is not complete anymore in $L_{2}(a, b)$ because (6) contains even functions only. Nevertheless considering the combined system of functions $\left\{f_{n}\right\}_{n=1}^{\infty} \cup\left\{g_{n}\right\}_{n=1}^{\infty}$ we obtain a complete system. The following theorem establishes that this remains true in a much more general situation.

Theorem 6 [18] Let $(a, b)$ be a finite interval and $f \in C^{2}(a, b) \cap C^{1}[a, b]$ be a complex valued function such that $f(x) \neq 0$ for any $x \in[a, b]$. Then the system of functions $\left\{f_{n}\right\}_{n=1}^{\infty} \cup\left\{g_{n}\right\}_{n=1}^{\infty}$ defined by the relations (5) and (11)-(3) with $x_{0}$ being an arbitrary point of the interval $[a, b]$ are complete in $L_{2}(a, b)$.

Proof. As in the proof of the previous theorem let us consider equation (14) where $q:=$ $-f^{\prime \prime} / f$, for example, with the boundary conditions (15). Due to theorem 2 any eigenfunction as well as any generalized eigenfunction of this problem can be represented as a uniformly convergent series in terms of the functions $f_{n}$ and $g_{n}$. From the completeness of the system of eigenfunctions and generalized eigenfunctions in $L_{2}(a, b)$ and by means of the Lauricella theorem we obtain the result.

Remark 7 If instead of the "seed" function $f$ one considers the function $1 / f$ then, as it is easy to see the auxiliary functions $\widetilde{X}^{(n)}$ and $X^{(n)}$ change their respective roles, and in the same way as was done above one can prove the completeness of the systems

$$
\left\{\frac{1}{f} X^{(2(n-1))}\right\}_{n=1}^{\infty} \quad \text { and } \quad\left\{\frac{1}{f} \widetilde{X}^{(2 n-1)}\right\}_{n=1}^{\infty}
$$

in $L_{2}(a, b)$ when $x_{0}$ coincides with $a$ or $b$ and the completeness of the union of these two systems in $L_{2}(a, b)$ when $x_{0}$ is an arbitrary point of the interval $[a, b]$. 
Remark 8 Under the considered conditions on the function $f$ it is easy to prove the completeness of the systems $\left\{X^{(2(n-1))}\right\}_{n=1}^{\infty},\left\{X^{(2 n-1)}\right\}_{n=1}^{\infty},\left\{\widetilde{X}^{(2 n-1)}\right\}_{n=1}^{\infty},\left\{\widetilde{X}^{(2(n-1))}\right\}_{n=1}^{\infty}$ when $x_{0}$ coincides with $a$ or $b$ and the completeness of the systems $\left\{\widetilde{X}^{(2(n-1))}\right\}_{n=1}^{\infty} \cup\left\{X^{(2 n-1)}\right\}_{n=1}^{\infty}$ and $\left\{\widetilde{X}^{(2 n-1)}\right\}_{n=1}^{\infty} \cup\left\{X^{(2(n-1))}\right\}_{n=1}^{\infty}$ when $x_{0}$ is an arbitrary point of the interval $[a, b]$. This is based on the previous theorems and on the observation that if $\left\{h_{n}\right\}_{n=1}^{\infty}$ is a complete system in $L_{2}(a, b)$ then taking a continuous in $[a, b]$ weight function $p$, such that $1 /|p|$ is separated from zero one has that $\left\{p h_{n}\right\}_{n=1}^{\infty}$ is complete in $L_{2}(a, b)$ as well. For the proof see [7, Sect. 4.77 .

Let us introduce the system of functions $\left\{\varphi_{k}\right\}_{k=0}^{\infty}$ defined as follows

$$
\varphi_{k}(x)= \begin{cases}f(x) X^{(k)}(x), & k \text { odd }, \\ f(x) \widetilde{X}^{(k)}(x), & k \text { even, }\end{cases}
$$

where the definition of $X^{(k)}$ and $\widetilde{X}^{(k)}$ is given by (10)-(3) with $x_{0}$ being an arbitrary point of the interval $[a, b]$. We are interested in the completeness of this system in the space of piecewise differentiable functions with respect to the maximum norm and in the corresponding series expansions.

The system of eigenfunctions of the Sturm-Liouville problem under certain regularity conditions is complete not only in the sense of the $L_{2}$-norm but also in the uniform convergence topology. The approach used in the proof of the previous two theorems and based on the completeness results for the system of eigenfunctions and generalized eigenfunctions of the Sturm-Liouville problem as well as on the Lauricella theorem about the transitivity of the property of completeness gives us the following result.

Theorem 9 Let $f$ satisfy the conditions of theorem [6 and $\left\{\varphi_{k}\right\}_{k=0}^{\infty}$ be the system of functions defined by (16) with $x_{0}$ being an arbitrary point of the interval $[a, b]$. Then for any complex valued piecewise continuously differentiable function $h$ defined on $[a, b]$ and for any $\varepsilon>0$ there exists such $N \in \mathbb{N}$ and such complex numbers $\alpha_{k}, k=0,1, \ldots N$ that $\max _{x \in[a, b]}\left|h(x)-\sum_{k=0}^{N} \alpha_{k} \varphi_{k}\right|<\varepsilon$.

Proof. Take a piecewise continuously differentiable function $h$. It satisfies certain boundary conditions of the form

$$
c_{1} h(a)+c_{2} h^{\prime}(a)=0 \quad \text { and } \quad c_{3} h(b)+c_{4} h^{\prime}(b)=0
$$

where $c_{1}^{2}+c_{2}^{2} \neq 0$ and $c_{3}^{2}+c_{4}^{2} \neq 0$. Let $\left\{u_{k}\right\}_{k=0}^{\infty}$ be a system of all eigenfunctions and generalized eigenfunctions of the Sturm-Liouville problem for equation (14) with the nondegenerate boundary conditions (17). This system is complete in the linear space of piecewise continuously differentiable functions with respect to the maximum norm (see 24, Chapter 1]). As was previously shown every $u_{k}$ admits a uniformly convergent series expansion in terms of the functions $\varphi_{k}$. Thus again, the result is a corollary of the Lauricella theorem.

Remark 10 In what follows instead of the system of functions $\left\{\varphi_{k}\right\}_{k=0}^{\infty}$ it will be slightly more convenient to consider the system $\left\{\psi_{k}=\frac{\varphi_{k}}{f}\right\}_{k=0}^{\infty}$ which from the previous theorem and due to the boundedness of $|f|$ and $1 /|f|$ is also complete with respect to the maximum norm in the space of piecewise continuously differentiable functions defined on $[a, b]$. 


\section{Generalized Taylor expansions}

In the previous section we showed that the system of recursive integrals

$$
\psi_{k}(x)=\left\{\begin{array}{cc}
X^{(k)}(x), & k \text { odd } \\
\widetilde{X}^{(k)}(x), & k \text { even }
\end{array}\right.
$$

is complete in $L_{2}(a, b)$ as well as with respect to the maximum norm in the space of piecewise differentiable functions defined on $[a, b]$. The system of recursive integrals generalizes the system of powers of the variable $x$. In this section we obtain generalizations of several classical results concerning the Taylor expansions. We start with a generalization of Taylor's formula.

Let us assume that the functions $f$ and $h$ on a certain segment $[a, b]$ possess the derivatives of all orders up to the order $n$ and that $f(x) \neq 0$, when $x \in[a, b]$. Then in $[a, b]$ the following generalized derivatives are defined

$$
\begin{gathered}
\gamma_{0}(h)(x)=h(x), \\
\gamma_{k}(h)(x)=\left(f^{2}(x)\right)^{(-1)^{k-1}}\left(\gamma_{k-1}(h)\right)^{\prime}(x),
\end{gathered}
$$

for $k=1,2, \ldots, n$. Let us consider a function of the form

$$
P_{n}(x)=\sum_{k=0}^{n} \alpha_{k} \psi_{k}(x) .
$$

Similarly to the fact that the coefficients of a polynomial $\sum_{k=0}^{n} a_{k}\left(x-x_{0}\right)^{k}$ can be expressed through its value and the values of its derivatives at the point $x_{0}$ we obtain that the coefficients $\alpha_{k}$ in (19) can be expressed through the value of $P_{n}$ and the values of its generalized derivatives at the point $x_{0}$. Indeed, a simple calculation gives us the following result

$$
\alpha_{k}=\frac{\gamma_{k}\left(P_{n}\right)\left(x_{0}\right)}{k !}
$$

Functions of the form (19) will be called generalized polynomials of order $n$. Notice that application of the generalized derivatives (18) to a generalized polynomial does not require the smoothness of $f,-$ formula (20) is true for any continuous and nonvanishing $f$.

Now let us consider a function $h$ possessing at the point $x_{0}$ the derivatives of all orders up to the order $n$ and suppose that the same is true for the function $f$ which additionally is different from zero at $x_{0}$. In relation with the function $h$ we introduce a generalized polynomial of the form (19) where the coefficients $\alpha_{k}$ are defined by the equality

$$
\alpha_{k}=\frac{\gamma_{k}(h)\left(x_{0}\right)}{k !} .
$$

According to the previous observation this generalized polynomial together with its generalized derivatives (up to the order $n$ ) at the point $x_{0}$ possess the same values as the function $h$ and its derivatives, $\gamma_{k}\left(P_{n}\right)\left(x_{0}\right)=\gamma_{k}(h)\left(x_{0}\right), k=0,1, \ldots n$. We are interested in estimating the difference between $P_{n}(x)$ and $h(x)$ for $x \neq x_{0}$. In the next theorem we obtain a generalization of the theorem on the Taylor remainder term in the Lagrange form. 
Theorem 11 (Generalized Taylor theorem with the Lagrange form of the remainder term) Let $\{f, h\} \subset C^{n+1}\left[x_{0}, b\right]$ and $f(x) \neq 0$ there. Then for any $x \in\left[x_{0}, b\right]$ there exists a number $c$ between $x_{0}$ and $x$ such that

$$
h(x)=\sum_{k=0}^{n} \frac{\gamma_{k}(h)\left(x_{0}\right)}{k !} \psi_{k}(x)+\frac{\gamma_{n+1}(h)(c)}{(n+1) !} \psi_{n+1}(x) .
$$

Proof. Consider the difference $R_{n}=h-P_{n}$ and the function $\psi_{n+1}$. For simplification of the notation let us skip the subindices, $R=R_{n}$ and $\psi=\psi_{n+1}$. We have that $\gamma_{k}(R)\left(x_{0}\right)=$ $\gamma_{k}(\psi)\left(x_{0}\right)=0, k=0,1, \ldots n$. We may therefore apply Cauchy's mean value theorem to the functions $R$ and $\psi$,

$$
\frac{R(x)}{\psi(x)}=\frac{R(x)-R\left(x_{0}\right)}{\psi(x)-\psi\left(x_{0}\right)}=\frac{R^{\prime}\left(x_{1}\right)}{\psi^{\prime}\left(x_{1}\right)}=\frac{f^{2}\left(x_{1}\right)}{f^{2}\left(x_{1}\right)} \frac{R^{\prime}\left(x_{1}\right)}{\psi^{\prime}\left(x_{1}\right)}=\frac{\gamma_{1}(R)\left(x_{1}\right)}{\gamma_{1}(\psi)\left(x_{1}\right)}
$$

where $x_{0}<x_{1}<x \leq b$. Another application of Cauchy's mean value theorem gives us the equalities

$$
\frac{\gamma_{1}(R)\left(x_{1}\right)}{\gamma_{1}(\psi)\left(x_{1}\right)}=\frac{\gamma_{1}(R)\left(x_{1}\right)-\gamma_{1}(R)\left(x_{0}\right)}{\gamma_{1}(\psi)\left(x_{1}\right)-\gamma_{1}(\psi)\left(x_{0}\right)}=\frac{\gamma_{1}^{\prime}(R)\left(x_{2}\right)}{\gamma_{1}^{\prime}(\psi)\left(x_{2}\right)}=\frac{f^{-2}\left(x_{2}\right)}{f^{-2}\left(x_{2}\right)} \frac{\gamma_{1}^{\prime}(R)\left(x_{2}\right)}{\gamma_{1}^{\prime}(\psi)\left(x_{2}\right)}=\frac{\gamma_{2}(R)\left(x_{2}\right)}{\gamma_{2}(\psi)\left(x_{2}\right)}
$$

where $x_{0}<x_{2}<x_{1}$. Continuing this procedure we obtain

$$
\begin{gathered}
\frac{\gamma_{n}(R)\left(x_{n}\right)}{\gamma_{n}(\psi)\left(x_{n}\right)}=\frac{\gamma_{n}(R)\left(x_{n}\right)-\gamma_{n}(R)\left(x_{0}\right)}{\gamma_{n}(\psi)\left(x_{n}\right)-\gamma_{n}(\psi)\left(x_{0}\right)}=\frac{\gamma_{n}^{\prime}(R)\left(x_{n+1}\right)}{\gamma_{n}^{\prime}(\psi)\left(x_{n+1}\right)} \\
=\frac{f^{(-1)^{n+1} 2}\left(x_{n+1}\right)}{f^{(-1)^{n+1} 2}\left(x_{n+1}\right)} \frac{\gamma_{n}^{\prime}(R)\left(x_{n+1}\right)}{\gamma_{n}^{\prime}(\psi)\left(x_{n+1}\right)}=\frac{\gamma_{n+1}(R)\left(x_{n+1}\right)}{\gamma_{n+1}(\psi)\left(x_{n+1}\right)}
\end{gathered}
$$

where $x_{0}<x_{n+1}<x_{n}<x_{n-1}<\cdots<x \leq b$. Consequently,

$$
\frac{R(x)}{\psi(x)}=\frac{\gamma_{n+1}(R)\left(x_{n+1}\right)}{\gamma_{n+1}(\psi)\left(x_{n+1}\right)}
$$

Moreover, by the definition of the functions $\psi_{k}$ and of the generalized derivatives we have that $\gamma_{n+1}(R)(x)=\gamma_{n+1}(h)(x)$ and $\gamma_{n+1}(\psi)(x)=(n+1)$ !. Then

$$
R(x)=\frac{\gamma_{n+1}(h)(c)}{(n+1) !} \psi(x),
$$

where $c=x_{n+1}$.

Obviously, the classical Taylor theorem with the Lagrange form of the remainder term is a special case of theorem 11 when $f \equiv 1$.

Definition 12 A functional series of the form

$$
\sum_{k=0}^{\infty} \frac{\gamma_{k}(h)\left(x_{0}\right)}{k !} \psi_{k}(x)
$$

will be called the generalized Taylor series of the function $h$. 
Theorem 2 gives us an important example of uniformly convergent generalized Taylor series. Indeed, we obtain that the quotients of the linearly independent solutions $u_{1}, u_{2}$ of equation (14) and the particular solution $f$ of (8) have the form

$$
\frac{u_{1}}{f}=\sum_{n=0}^{\infty} \frac{1+(-1)^{n}}{2 n !} \lambda^{\frac{n}{2}} \psi_{n} \quad \text { and } \quad \frac{u_{2}}{f}=\sum_{n=0}^{\infty} \frac{1+(-1)^{n+1}}{2 n !} \lambda^{\frac{n-1}{2}} \psi_{n} .
$$

\section{A relation between the generalized and the classical Taylor expansions}

In this section we establish relations between the generalized Taylor coefficients of a sufficiently smooth function and its usual Taylor coefficients.

Theorem 13 Let $\{f, h\} \subset C^{n}[a, b]$ and $f(x) \neq 0, x \in[a, b]$ and $\varphi=f^{2}$. The following relation between the ordinary derivatives and generalized derivatives of the function $h$ at the point $x_{0} \in[a, b]$ are valid

$$
\left(\begin{array}{c}
h\left(x_{0}\right) \\
h^{\prime}\left(x_{0}\right) \\
h^{\prime \prime}\left(x_{0}\right) \\
h^{\prime \prime \prime}\left(x_{0}\right) \\
\vdots \\
h^{[n]}\left(x_{0}\right)
\end{array}\right)=\left(\begin{array}{cccccc}
1 & 0 & 0 & 0 & \ldots & 0 \\
0 & 1 / \varphi\left(x_{0}\right) & 0 & 0 & \ldots & 0 \\
0 & a_{2,1}\left(x_{0}\right) & 1 & 0 & \ldots & 0 \\
0 & a_{3,1}\left(x_{0}\right) & a_{3,2}\left(x_{0}\right) & 1 / \varphi\left(x_{0}\right) & \ldots & 0 \\
\vdots & \vdots & \vdots & \vdots & \ddots & \vdots \\
0 & a_{n, 1}\left(x_{0}\right) & a_{n, 2}\left(x_{0}\right) & a_{n, 3}\left(x_{0}\right) & \ldots & \varphi^{-\frac{1+(-1)^{n+1}}{2}}\left(x_{0}\right)
\end{array}\right)\left(\begin{array}{c}
\gamma_{0}(h)\left(x_{0}\right) \\
\gamma_{1}(h)\left(x_{0}\right) \\
\gamma_{2}(h)\left(x_{0}\right) \\
\gamma_{3}(h)\left(x_{0}\right) \\
\vdots \\
\gamma_{n}(h)\left(x_{0}\right)
\end{array}\right)
$$

where the functions $a_{n, m}$ are calculated following the recursive procedure:

$$
a_{n, m}=a_{n-1, m}^{\prime}+\varphi^{(-1)^{m}} a_{n-1, m-1} .
$$

Proof. The first two rows in (22) we obtain directly from the definition of the generalized derivatives. To obtain $h^{\prime \prime}$ we proceed in the following way

$$
h^{\prime \prime}=\left(\frac{\gamma_{1}(h)}{\varphi}\right)^{\prime}=\frac{1}{\varphi}\left(\gamma_{1}(h)\right)^{\prime}+\left(\frac{1}{\varphi}\right)^{\prime} \gamma_{1}(h) .
$$

From (18) we have

$$
\left(\gamma_{k}(h)\right)^{\prime}=\varphi^{(-1)^{k+1}} \gamma_{k+1}(h) .
$$

In particular, $\left(\gamma_{1}(h)\right)^{\prime}=\varphi \gamma_{2}(h)$. Thus, $h^{\prime \prime}=\gamma_{2}(h)+\left(\frac{1}{\varphi}\right)^{\prime} \gamma_{1}(h)$ (and hence $\left.a_{2,1}=\left(\frac{1}{\varphi}\right)^{\prime}\right)$.

In general, assume that we have calculated the row corresponding to $h^{[k]}$ where $k$ is even,

$$
h^{[k]}=\gamma_{k}(h)+a_{k, k-1} \gamma_{k-1}(h)+\cdots+a_{k, 1} \gamma_{1}(h) .
$$

Differentiating this equality we obtain

$$
h^{[k+1]}=\left(\gamma_{k}(h)\right)^{\prime}+a_{k, k-1}\left(\gamma_{k-1}(h)\right)^{\prime}+\cdots+a_{k, 1}\left(\gamma_{1}(h)\right)^{\prime}+a_{k, k-1}^{\prime} \gamma_{k-1}(h)+\cdots+a_{k, 1}^{\prime} \gamma_{1}(h) .
$$


Replacing the derivatives of $\gamma_{j}(h), j=1, \ldots, k$ with $\varphi^{(-1)^{j+1}} \gamma_{j+1}(h)$ we arrive at (23) . For an odd $k$ the reasoning is analogous.

The transformation matrix in (22) will be denoted by $A_{n}$.

Meanwhile the recursive procedure (23) allows one to calculate the first few rows of the transformation matrix $A_{n}$ the next statement gives us a general formula for its elements.

Proposition 14 The element $a_{n, m}$ with $1 \leq n \leq N$ and $2 \leq m \leq n$ of the matrix $A_{N}$, $N=2,3, \ldots$ in (22) has the form

$$
a_{n, m}=\sum_{k=m-1}^{n-1}\left(\begin{array}{c}
n-1 \\
k
\end{array}\right)\left(\frac{1}{\varphi}\right)^{[n-1-k]} b_{k, m-1}
$$

where $\left(\begin{array}{l}p \\ q\end{array}\right)$ represents the binomial coefficients,

$$
\begin{aligned}
b_{k, m} & =\sum_{k_{1}=m-1}^{k-1}\left(\begin{array}{c}
k-1 \\
k_{1}
\end{array}\right) \varphi^{\left[k-1-k_{1}\right]} \sum_{k_{2}=m-2}^{k_{1}-1}\left(\begin{array}{c}
k_{1}-1 \\
k_{2}
\end{array}\right)\left(\frac{1}{\varphi}\right)^{\left[k_{1}-1-k_{2}\right]} \ldots \\
& \sum_{k_{m-2}=2}^{k_{m-3}-1}\left(\begin{array}{c}
k_{m-3}-1 \\
k_{m-2}
\end{array}\right)\left(\frac{1}{\varphi}\right)^{\left[k_{m-3}-1-k_{m-2}\right]} \sum_{k_{m-1}=1}^{k_{m-2}-1}\left(\begin{array}{c}
k_{m-2}-1 \\
k_{m-1}
\end{array}\right) \varphi^{\left[k_{m-2}-1-k_{m-1}\right]}\left(\frac{1}{\varphi}\right)^{\left[k_{m-1}-1\right]}
\end{aligned}
$$

when $m$ is even and

$$
\begin{aligned}
b_{k, m} & =\sum_{k_{1}=m-1}^{k-1}\left(\begin{array}{c}
k-1 \\
k_{1}
\end{array}\right) \varphi^{\left[k-1-k_{1}\right]} \sum_{k_{2}=m-2}^{k_{1}-1}\left(\begin{array}{c}
k_{1}-1 \\
k_{2}
\end{array}\right)\left(\frac{1}{\varphi}\right)^{\left[k_{1}-1-k_{2}\right]} \ldots \\
& \sum_{k_{m-2}=2}^{k_{m-3}-1}\left(\begin{array}{c}
k_{m-3}-1 \\
k_{m-2}
\end{array}\right) \varphi^{\left[k_{m-3}-1-k_{m-2}\right]} \sum_{k_{m-1}=1}^{k_{m-2}-1}\left(\begin{array}{c}
k_{m-2}-1 \\
k_{m-1}
\end{array}\right)\left(\frac{1}{\varphi}\right)^{\left[k_{m-2}-1-k_{m-1}\right]} \varphi^{\left[k_{m-1}-1\right]}
\end{aligned}
$$

when $m$ is odd (in both cases, $2 \leq m \leq k$ ).

Proof. Let us notice that

$$
h^{\prime}=\frac{1}{\varphi} \gamma_{1}
$$

(we will write $\gamma_{k}$ understanding $\left.\gamma_{k}(h)\right)$ and recall the formula for the $n$-th derivative of a product of two functions

$$
(f g)^{[n]}=\sum_{k=0}^{n}\left(\begin{array}{l}
n \\
k
\end{array}\right) f^{[n-k]} g^{[k]} .
$$

Applying this formula in order to evaluate the $(n-1)$-th derivative of the equality (25) we obtain

$$
h^{[n]}=\sum_{k=0}^{n-1}\left(\begin{array}{c}
n-1 \\
k
\end{array}\right)\left(\frac{1}{\varphi}\right)^{[n-1-k]} \gamma_{1}^{[k]}, \quad n \geq 1 .
$$


Considering $\gamma_{1}^{[k]}, k \geq 1$ we obtain

$$
\begin{aligned}
& \gamma_{1}^{[k]}=\sum_{k_{1}=0}^{k-1}\left(\begin{array}{c}
k-1 \\
k_{1}
\end{array}\right) \varphi^{\left[k-1-k_{1}\right]} \gamma_{2}^{\left[k_{1}\right]} \\
& =\varphi^{[k-1]} \gamma_{2}+\sum_{k_{1}=1}^{k-1}\left(\begin{array}{c}
k-1 \\
k_{1}
\end{array}\right) \varphi^{\left[k-1-k_{1}\right]} \gamma_{2}^{\left[k_{1}\right]} \\
& =\varphi^{[k-1]} \gamma_{2}+\sum_{k_{1}=1}^{k-1}\left(\begin{array}{c}
k-1 \\
k_{1}
\end{array}\right) \varphi^{\left[k-1-k_{1}\right]} \sum_{k_{2}=0}^{k_{1}-1}\left(\begin{array}{c}
k_{1}-1 \\
k_{2}
\end{array}\right)\left(\frac{1}{\varphi}\right)^{\left[k_{1}-1-k_{2}\right]} \gamma_{3}^{\left[k_{2}\right]} \\
& =\varphi^{[k-1]} \gamma_{2}+\sum_{k_{1}=1}^{k-1}\left(\begin{array}{c}
k-1 \\
k_{1}
\end{array}\right) \varphi^{\left[k-1-k_{1}\right]}\left(\frac{1}{\varphi}\right)^{\left[k_{1}-1\right]} \gamma_{3}+ \\
& +\sum_{k_{1}=2}^{k-1}\left(\begin{array}{c}
k-1 \\
k_{1}
\end{array}\right) \varphi^{\left[k-1-k_{1}\right]} \sum_{k_{2}=1}^{k_{1}-1}\left(\begin{array}{c}
k_{1}-1 \\
k_{2}
\end{array}\right)\left(\frac{1}{\varphi}\right)^{\left[k_{1}-1-k_{2}\right]} \gamma_{3}^{\left[k_{2}\right]} \\
& =\varphi^{[k-1]} \gamma_{2}+\sum_{k_{1}=1}^{k-1}\left(\begin{array}{c}
k-1 \\
k_{1}
\end{array}\right) \varphi^{\left[k-1-k_{1}\right]}\left(\frac{1}{\varphi}\right)^{\left[k_{1}-1\right]} \gamma_{3}+ \\
& \sum_{k_{1}=2}^{k-1}\left(\begin{array}{c}
k-1 \\
k_{1}
\end{array}\right) \varphi^{\left[k-1-k_{1}\right]} \sum_{k_{2}=1}^{k_{1}-1}\left(\begin{array}{c}
k_{1}-1 \\
k_{2}
\end{array}\right)\left(\frac{1}{\varphi}\right)^{\left[k_{1}-1-k_{2}\right]} \sum_{k_{3}=0}^{k_{2}-1}\left(\begin{array}{c}
k_{2}-1 \\
k_{3}
\end{array}\right) \varphi^{\left[k_{2}-1-k_{3}\right]} \gamma_{4}^{\left[k_{3}\right]} \\
& =\varphi^{[k-1]} \gamma_{2}+\sum_{k_{1}=1}^{k-1}\left(\begin{array}{c}
k-1 \\
k_{1}
\end{array}\right) \varphi^{\left[k-1-k_{1}\right]}\left(\frac{1}{\varphi}\right)^{\left[k_{1}-1\right]} \gamma_{3}+ \\
& \sum_{k_{1}=2}^{k-1}\left(\begin{array}{c}
k-1 \\
k_{1}
\end{array}\right) \varphi^{\left[k-1-k_{1}\right]} \sum_{k_{2}=1}^{k_{1}-1}\left(\begin{array}{c}
k_{1}-1 \\
k_{2}
\end{array}\right)\left(\frac{1}{\varphi}\right)^{\left[k_{1}-1-k_{2}\right]} \varphi^{\left[k_{2}-1\right]} \gamma_{4}+ \\
& \sum_{k_{1}=3}^{k-1}\left(\begin{array}{c}
k-1 \\
k_{1}
\end{array}\right) \varphi^{\left[k-1-k_{1}\right]} \sum_{k_{2}=2}^{k_{1}-1}\left(\begin{array}{c}
k_{1}-1 \\
k_{2}
\end{array}\right)\left(\frac{1}{\varphi}\right)^{\left[k_{1}-1-k_{2}\right]} \sum_{k_{3}=1}^{k_{2}-1}\left(\begin{array}{c}
k_{2}-1 \\
k_{3}
\end{array}\right) \varphi^{\left[k_{2}-1-k_{3}\right]} \gamma_{4}^{\left[k_{3}\right]} \text {. }
\end{aligned}
$$

Continuing this procedure we have

$$
\gamma_{1}^{[k]}=b_{k, 1} \gamma_{2}+b_{k, 2} \gamma_{3}+b_{k, 3} \gamma_{4}+\cdots+b_{k, k} \gamma_{k+1}
$$

where

$$
\begin{gathered}
b_{k, 1}=\varphi^{[k-1]} \\
b_{k, 2}=\sum_{k_{1}=1}^{k-1}\left(\begin{array}{c}
k-1 \\
k_{1}
\end{array}\right) \varphi^{\left[k-1-k_{1}\right]}\left(\frac{1}{\varphi}\right)^{\left[k_{1}-1\right]} \\
b_{k, 3}=\sum_{k_{1}=2}^{k-1}\left(\begin{array}{c}
k-1 \\
k_{1}
\end{array}\right) \varphi^{\left[k-1-k_{1}\right]} \sum_{k_{2}=1}^{k_{1}-1}\left(\begin{array}{c}
k_{1}-1 \\
k_{2}
\end{array}\right)\left(\frac{1}{\varphi}\right)^{\left[k_{1}-1-k_{2}\right]} \varphi^{\left[k_{2}-1\right]} \\
b_{k, 4}=\sum_{k_{1}=3}^{k-1}\left(\begin{array}{c}
k-1 \\
k_{1}
\end{array}\right) \varphi^{\left[k-1-k_{1}\right]} \sum_{k_{2}=2}^{k_{1}-1}\left(\begin{array}{c}
k_{1}-1 \\
k_{2}
\end{array}\right)\left(\frac{1}{\varphi}\right)^{\left[k_{1}-1-k_{2}\right]} \sum_{k_{3}=1}^{k_{2}-1}\left(\begin{array}{c}
k_{2}-1 \\
k_{3}
\end{array}\right) \varphi^{\left[k_{2}-1-k_{3}\right]}\left(\frac{1}{\varphi}\right)^{\left[k_{3}-1\right]}
\end{gathered}
$$


and in general $b_{k, m}$ are defined by the equalities from the statement of the proposition.

From (26) we obtain for $n \geq 1$

$$
\begin{aligned}
h^{[n]} & =\sum_{k=0}^{n-1}\left(\begin{array}{c}
n-1 \\
k
\end{array}\right)\left(\frac{1}{\varphi}\right)^{[n-1-k]} \gamma_{1}^{[k]} \\
& =\left(\frac{1}{\varphi}\right)^{[n-1]} \gamma_{1}+\sum_{k=1}^{n-1}\left(\begin{array}{c}
n-1 \\
k
\end{array}\right)\left(\frac{1}{\varphi}\right)^{[n-1-k]} \gamma_{1}^{[k]} \\
& =\left(\frac{1}{\varphi}\right)^{[n-1]} \gamma_{1}+\sum_{k=1}^{n-1}\left(\begin{array}{c}
n-1 \\
k
\end{array}\right)\left(\frac{1}{\varphi}\right)^{[n-1-k]} \sum_{m=1}^{k} b_{k, m} \gamma_{m+1} \\
& =a_{n, 1} \gamma_{1}+a_{n, 2} \gamma_{2}+a_{n, 3} \gamma_{3}+a_{n, 4} \gamma_{4}+\cdots+a_{n, n-1} \gamma_{n-1}+a_{n, n} \gamma_{n}
\end{aligned}
$$

where

$$
\begin{gathered}
a_{n, 1}=\left(\frac{1}{\varphi}\right)^{[n-1]} \\
a_{n, 2}=\sum_{k=1}^{n-1}\left(\begin{array}{c}
n-1 \\
k
\end{array}\right)\left(\frac{1}{\varphi}\right)^{[n-1-k]} b_{k, 1} \\
a_{n, 3}=\sum_{k=2}^{n-1}\left(\begin{array}{c}
n-1 \\
k
\end{array}\right)\left(\frac{1}{\varphi}\right)^{[n-1-k]} b_{k, 2} \\
a_{n, 4}=\sum_{k=3}^{n-1}\left(\begin{array}{c}
n-1 \\
k
\end{array}\right)\left(\frac{1}{\varphi}\right)^{[n-1-k]} b_{k, 3} \\
a_{n, 5}=\sum_{k=4}^{n-1}\left(\begin{array}{c}
n-1 \\
k
\end{array}\right)\left(\frac{1}{\varphi}\right)^{[n-1-k]} b_{k, 4} \\
a_{n, n-1}=\sum_{k=n-2}^{n-1}\left(\begin{array}{c}
n-1 \\
k
\end{array}\right)\left(\frac{1}{\varphi}\right)^{[n-1-k]} b_{k, n-2} \\
a_{n, n}=\sum_{k=n-1}\left(\begin{array}{c}
n-1 \\
k
\end{array}\right)\left(\frac{1}{\varphi}\right)^{[n-1-k]} b_{k, n-1}
\end{gathered}
$$

and hence

$$
a_{n, m}=\sum_{k=m-1}^{n-1}\left(\begin{array}{c}
n-1 \\
k
\end{array}\right)\left(\frac{1}{\varphi}\right)^{[n-1-k]} b_{k, m-1}, \quad 2 \leq m \leq n
$$




\section{Taylor coefficients of solutions of the Sturm-Liouville equa- tion}

In the present section we obtain an interesting corollary of theorem 13, Given a nonvanishing (complex valued) solution of equation (10) possessing $n$ derivatives at a certain point, for any solution of (14) (for any value of the spectral parameter $\lambda$ ) we are able to calculate the exact values of its $n$ derivatives at the same point without any integration. Moreover, each derivative is a polynomial of a certain order with respect to $\lambda$.

Theorem 15 Let $(a, b)$ be a finite interval and $f \in C^{2}(a, b) \cap C^{1}[a, b]$ be a complex valued solution of (10) such that $f(x) \neq 0$ for any $x \in[a, b]$ and at the point $x_{0} \in[a, b]$ there exist the derivatives of $f$ up to the $n$-th order. Then the linearly independent solutions $u_{1}$ and $u_{2}$ of equation (14) satisfying the initial conditions (12) and (13) respectively possess at the point $x_{0}$ the derivatives up to the $n$-th order which can be calculated according to the following relations

$$
\left(\begin{array}{c}
u_{1}\left(x_{0}\right) / f\left(x_{0}\right) \\
\left(u_{1} / f\right)^{\prime}\left(x_{0}\right) \\
\left(u_{1} / f\right)^{\prime \prime}\left(x_{0}\right) \\
\left(u_{1} / f\right)^{\prime \prime \prime}\left(x_{0}\right) \\
\left(u_{1} / f\right)^{I V}\left(x_{0}\right) \\
\vdots \\
\left(u_{1} / f\right)^{[n]}\left(x_{0}\right)
\end{array}\right)=A_{n}\left(\begin{array}{c}
1 \\
0 \\
\lambda \\
0 \\
\lambda^{2} \\
\vdots \\
\frac{1+(-1)^{n}}{2} \lambda^{\frac{n}{2}}
\end{array}\right)
$$

and

$$
\left(\begin{array}{c}
u_{2}\left(x_{0}\right) / f\left(x_{0}\right) \\
\left(u_{2} / f\right)^{\prime}\left(x_{0}\right) \\
\left(u_{2} / f\right)^{\prime \prime}\left(x_{0}\right) \\
\left(u_{2} / f\right)^{\prime \prime \prime}\left(x_{0}\right) \\
\left(u_{2} / f\right)^{I V}\left(x_{0}\right) \\
\vdots \\
\left(u_{2} / f\right)^{[n]}\left(x_{0}\right)
\end{array}\right)=A_{n}\left(\begin{array}{c}
0 \\
1 \\
0 \\
\lambda \\
0 \\
\vdots \\
\frac{1+(-1)^{n+1}}{2} \lambda^{\frac{n-1}{2}}
\end{array}\right)
$$

where $A_{n}$ is the matrix from (22).

Proof. From theorem 2 we have that in $(a, b)$ the functions $u_{1}$ and $u_{2}$ defined by (11) are solutions of the Sturm-Liouville equation (14) satisfying the initial conditions (12) and (13), and the series in (11) converge uniformly on $[a, b]$. Thus, the functions $u_{1} / f$ and $u_{2} / f$ are expended into uniformly convergent generalized Taylor series, and from (11) we have that

$$
\gamma_{k}\left(\frac{u_{1}}{f}\right)\left(x_{0}\right)=\frac{1+(-1)^{k}}{2} \lambda^{\frac{k}{2}}
$$

and

$$
\gamma_{k}\left(\frac{u_{2}}{f}\right)\left(x_{0}\right)=\frac{1+(-1)^{k+1}}{2} \lambda^{\frac{k-1}{2}}
$$


Example 16 Our first example is the equation (14) with a constant coefficient $q=-c^{2}, c \in$ $\mathbb{R}, c \neq 0$. Obviously, a nonvanishing solution of (10) can be chosen in the form $f(x)=e^{c x}$. Considering $x_{0}=0$ we calculate the first six rows of the matrix $A_{n}$. We have

$$
A_{5}=\left(\begin{array}{cccccc}
1 & 0 & 0 & 0 & 0 & 0 \\
0 & 1 & 0 & 0 & 0 & 0 \\
0 & -2 c & 1 & 0 & 0 & 0 \\
0 & 4 c^{2} & -2 c & 1 & 0 & 0 \\
0 & -8 c^{3} & 4 c^{2} & -4 c & 1 & 0 \\
0 & 16 c^{4} & -8 c^{3} & 12 c^{2} & -4 c & 1
\end{array}\right) .
$$

On the other hand it is easy to see that the solution $u_{1}$ from (11) in this case has the form

$$
u_{1}(x)=\frac{c+\kappa}{2 \kappa} e^{\kappa x}+\frac{\kappa-c}{2 \kappa} e^{-\kappa x},
$$

where $\kappa=\sqrt{c^{2}+\lambda}$. Simple calculation involving the quotient $u_{1} / f=\frac{c+\kappa}{2 \kappa} e^{(\kappa-c) x}+\frac{\kappa-c}{2 \kappa} e^{-(\kappa+c) x}$ gives us the following values

$$
\begin{gathered}
\left(u_{1} / f\right)^{\prime}(0)=0, \quad\left(u_{1} / f\right)^{\prime \prime}(0)=\lambda, \quad\left(u_{1} / f\right)^{\prime \prime \prime}(0)=-2 c \lambda, \\
\left(u_{1} / f\right)^{I V}(0)=4 c^{2} \lambda+\lambda^{2}, \quad\left(u_{1} / f\right)^{V}(0)=-8 c^{3} \lambda-4 c \lambda^{2}
\end{gathered}
$$

which is exactly the result of multiplication of the matrix $A_{5}$ by the vector

$$
\left(1,0, \lambda, 0, \lambda^{2}, 0\right)^{\top}
$$

(see (27)).

Example 17 Consider the equation

$$
u^{\prime \prime}(x)-a^{2} x^{2 k} u(x)=\lambda u(x) .
$$

This equation with $\lambda=-1$ can be found, e.g., in [10, Part 3, Chapter II, eq. 2.15] where it is stated that its solution is unknown, meanwhile the solution of (28) when $\lambda=0$ is known [10, Part 3, Chapter II, eq. 2.14] even for noninteger $k$. In particular, when $k=-\frac{2 n}{2 n+1}$ and $n$ is a negative integer number the general solution of the equation

$$
y^{\prime \prime}(x)-a^{2} x^{2 k} y(x)=0
$$

has the form

$$
y(x)=\left(x^{\frac{2 n-1}{2 n+1}} \frac{d}{d x}\right)^{-n}\left(c_{1} \exp \left(a(2 n+1) x^{\frac{1}{2 n+1}}\right)+c_{2} \exp \left(-a(2 n+1) x^{\frac{1}{2 n+1}}\right)\right) .
$$

This solution can be used for solving (28). Consider, for example, $k=-2(n=-1)$. The general solution of (29) takes the form

$$
y(x)=a x\left(c_{1} e^{-a / x}-c_{2} e^{a / x}\right) .
$$


As a particular solution $f$ we can take, e.g., $f(x)=a x e^{a / x}$. Fix $x_{0}=1$. Calculation of the first five rows of the matrix $A_{n}$ gives us the following result

$$
A_{4}=\left(\begin{array}{ccccc}
1 & 0 & 0 & 0 & 0 \\
0 & \frac{e^{-2 a}}{a^{2}} & 0 & 0 & 0 \\
0 & \frac{2(a-1) e^{-2 a}}{a^{2}} & 1 & 0 & 0 \\
0 & e^{-2 a}\left(4+\frac{6}{a^{2}}(1-2 a)\right) & 2(a-1) & \frac{e^{-2 a}}{a^{2}} & 0 \\
0 & e^{-2 a\left(24-16 a-\frac{24(1-a)}{a^{2}}\right)} & 8-16 a+4 a^{2} & \frac{4(a-1)}{a^{2}} e^{-2 a} & 1
\end{array}\right)
$$

from where using theorem 15 the first four derivatives of the functions $u_{1} / f$ and $u_{2} / f$ at $x_{0}=1$ can be calculated.

\section{Conclusions}

A formula for calculating Taylor coefficients of the solution of the Sturm-Liouville equation is obtained. It is based on the knowledge of the Taylor coefficients of a particular solution of the equation corresponding to the zero value of a spectral parameter. The form of the (matrix) map transforming one set of Taylor coefficients into the other is independent of the particular form of the particular solution.

\section{Acknowledgements}

Research of V.K. was supported by CONACYT, Mexico via the research project 50424. S.T. wants to thank the Department of Mathematics of the Cinvestav, campus Queretaro for the hospitality during a part of his sabbatical year. The research of S.T. is partly supported by grant from NSERC of Canada.

\section{References}

[1] H. Begehr and R. Gilbert, Transformations, transmutations and kernel functions, vol. 1-2. Longman, Pitman, 1992.

[2] L. Bers, Theory of pseudo-analytic functions, New York University, 1952.

[3] H. Campos and V.V. Kravchenko, A finite-sum representation for solutions for the Jacobi operator, Journal of Difference Equations and Applications 17 No. 42011 567-575.

[4] R. Carroll, Transmutation theory and applications. Amsterdam: North-Holland, 1986.

[5] R. Castillo-Pérez, K.V. Khmelnytskaya, V.V. Kravchenko and H. Oviedo, Efficient calculation of the reflectance and transmittance of finite inhomogeneous layers, Journal of Optics A: Pure and Applied Optics 11 No. 62009065707.

[6] R. Castillo-Pérez, V.V. Kravchenko, H. Oviedo and V.S. Rabinovich, Dispersion equation and eigenvalues for quantum wells using spectral parameter power series. To appear in Journal of Mathematical Physics. 
[7] L. Collatz, Functional analysis and computational mathematics, Moscow: Mir, 1969 (russian translation of the german edition Collatz L Funktionalanalysis und Numerische Mathematik, Berlin: Springer-Verlag, 1964).

[8] P.J. Davis, Interpolation and Approximation, Dover Publications, 1975.

[9] M.K. Fage, N.I. Nagnibida, The problem of equivalence of ordinary linear differential operators, Novosibirsk: Nauka, 1987 (in Russian).

[10] E. Kamke, Handbook of ordinary differential equations, Moscow: Nauka, 1976 (Russian translation from the German original: Differentialgleichungen, Lösungsmethoden und Lösungen, Leipzig, 1959).

[11] K.V. Khmelnytskaya and H.C. Rosu, An amplitude-phase (Ermakov-Lewis) approach for the Jackiw-Pi model of bilayer graphene, J. of Phys. A 42 issue 42009042004.

[12] K.V. Khmelnytskaya and H.C. Rosu, A new series representation for Hill's discriminant. Annals of Physics 325 issue 112010 2512-2521.

[13] K.V. Khmelnytskaya and H.C. Rosu, Bloch solutions of periodic Dirac equations in SPPS form. To appear in the Proc. of IWOTA 2009, available from arXiv:1006.3596.

[14] K.V. Khmelnytskaya, H.C. Rosu and A. Gonzalez, Periodic Sturm-Liouville problems related to two Riccati equations of constant coefficients, Annals of Physics v. 33 issue 42010 469-472.

[15] K.V. Khmelnytskaya and T.V. Torchynska, Reconstruction of potentials in quantum dots and other small symmetric structures, Mathematical Methods in the Applied Sciences v. 33 issue 4 $2010469-472$.

[16] V.V. Kravchenko, A representation for solutions of the Sturm-Liouville equation, Complex Variables and Elliptic Problems v. 53 issue 82008 775-789.

[17] V.V. Kravchenko, Applied Pseudoanalytic Function Theory, Basel: Birkhäuser, Series: Frontiers in Mathematics, 2009.

[18] V.V. Kravchenko, On the completeness of systems of recursive integrals, Communications in Mathematical Analysis, Conf. 032011 172-176.

[19] V.V. Kravchenko and R.M. Porter, Spectral parameter power series for Sturm-Liouville problems, Mathematical Methods in the Applied Sciences v. 33 issue 42010 459-468.

[20] V.V. Kravchenko and R.M. Porter, Conformal Mapping of Right Circular Quadrilaterals, Complex Variables and Elliptic Equations, First Published on: 08 July 2010. DOI: 10.1080/17476930903276100.

[21] V.V. Kravchenko and U. Velasco-García, Dispersion equation and eigenvalues for the ZakharovShabat system using spectral parameter power series. Submitted.

[22] B.M. Levitan Inverse Sturm-Liouville problems. VSP, Zeist, 1987.

[23] B.M. Levitan and I.S. Sargsjan, Sturm-Liouville and Dirac operators, Dordrecht: Kluwer Acad. Publ., 1991. 
[24] V.A. Marchenko, Sturm-Liouville operators and applications, Basel: Birkhäuser, 1986.

[25] S.M. Sitnik, Transmutations and applications: a survey. arXiv:1012.3741v1 [math.CA], originally published in the book: "Advances in Modern Analysis and Mathematical Modeling" Editors: Yu.F.Korobeinik, A.G.Kusraev, Vladikavkaz: Vladikavkaz Scientific Center of the Russian Academy of Sciences and Republic of North Ossetia-Alania, 2008, 226-293.

[26] K. Trimeche Transmutation operators and mean-periodic functions associated with differential operators, London: Harwood Academic Publishers, 1988. 